\title{
An abutment screw loosening study of a Diamond Like Carbon-coated CP titanium implant
}

\author{
S. K. KIM*, J. B. LEE*, J. Y. KOAK*, S. J. HEO*, K. R. $\mathrm{LEE}^{\dagger}, \mathrm{L} \cdot \mathrm{R} \cdot \mathrm{CHO}^{\ddagger}$ \& \\ S. S. LEE ${ }^{\S}{ }^{*}$ Department of Prosthodontics and Dental Research Institute, College of Dentistry, Seoul National University, ${ }^{\dagger}$ Thin Film \\ Technology Research Center, Korea Institute of Science and Technology, Seoul, ${ }^{\ddagger}$ Department of Prosthodontics, College of Dentistry, Kangnung \\ National University and ${ }^{\S}$ Department of Oral and Maxillofacial Radiology, College of Dentistry, Seoul National University, Korea
}

SUMMARY The aim of this study was to quantify the extent of abutment screw loosening and thus understand the role of frictional and wear factors in abutment screw loosening by using a cyclic loading device to compare Diamond Like Carbon (DLC)-coated and non-coated implants. The properties of DLC films, including hardness, wear resistance, chemical stability, and biocompatibility, are similar to those of real diamond materials. In this study, a $1-\mu \mathrm{m}$ thick DLC film served to protect and lubricate a layer of commercially-pure titanium affixed to the top of a dental implant (external hexagon-shaped implant). A cyclic loading force was then applied to the top of the prosthetic portion of the implants in order to determine the difference in looseness of the titanium abutment screw between ten DLC-coated implants and ten non-coated implants. The abutment screw loosening tests were performed with $100 \mathrm{~N}$ of force at a frequency of $20 \mathrm{~Hz}$. Data indicate that implants with a DLC coating are more resistant to an applied force $(P=0.002)$ than are those without the coating. We hope these results will be useful for preventing implant abutment screw loosening.

KEYWORDS: implant, wear resistance, abutment screw loosening, Diamond Like Carbon, cyclic loading, coating

Accepted for publication 18 October 2004

\section{Introduction}

In recent decades, dental implants have been increasingly used for edentulous patients. Various problems have arisen with this approach, and, in particular, there is potential for the prosthesis to loosen in single tooth restorations employing screw-retained implants (1). Consequently, several anti-rotational features have been developed to prevent screw loosening.

For the continuing use of an implant, it is important that the contact surface of the implant and the opposing bearing surface of the abutment remain stable throughout the life of the prosthesis. The preload stress achieved in the screw tightening procedure is also a critical factor in abutment screw loosening (2). Apart from the preload stress achieved in screw tightening, the stability of a screw joint is believed to be a function of the geometry of the screw, the contact between the screw head and the abutment screw bore, the material construction of the implant, and the friction between the various implant parts $(2,3)$. Among these factors, the present study focused on friction, particularly at the top of the implants, as a cause of screw loosening.

Materials with special characteristics, in particular those that are lightweight, strong, durable and resistant to wear, are very useful as anti-frictional agents. From a medical-mechanical perspective, several such materials exist: titanium alloys $\left(\mathrm{Ti}_{6} \mathrm{Al}_{4} \mathrm{~V}\right)$, alumina ceramics $\left(\mathrm{Al}_{2} \mathrm{O}_{3}\right)$, and composites such as TiN coating on graphite. These intriguing materials present several drawbacks in dental applications, however, and have thus motivated a great deal of work aimed at improving the properties of more traditional biomaterials. 
Dentistry, and especially implants and removable appliance frameworks, have seen frequent uses for titanium as a biomaterial because it forms an electrically- and chemically-inert passivated layer on the surface of the bone and has good biocompatibility with same (4). In the oral environment, however, commercially-pure (c.p.) titanium has inferior qualities in terms of hardness, either when mechanically-irritated or upon accumulation of plaque. To avoid this problem, titanium is generally coated with a nitride or epoxy, and these coatings do have some success in preventing biodegradation $(5,6)$. Diamond Like Carbon (DLC) or a crystalline diamond coating can also be used to increase the wear resistance of, among other materials, titanium (7).

A DLC film has properties similar to those of real diamond, including hardness, wear resistance, and chemical stability (8). Moreover, low friction resistance and excellent wear resistance make DLC one of the best materials for use as a protectant and lubricant. Such applications have been found in computer hard disks, VCR head drums, and some friction-bearing materials. Among the many favourable properties of DLC films, the most valuable for dentistry is its similar biocompatibility to titanium and 316 stainless steel (8).

Before DLC can be accepted into common use, however, the stability of the prosthesis containing this film must be prudently and thoroughly evaluated in clinical as well as laboratory trials (9). Accordingly, our study aimed to evaluate the effectiveness of a DLC coating on the top of an implant's external hexagon when subjected to cyclic loading.

\section{Materials and methods}

\section{DLC coating}

Twenty self-tapping external hexagon type implants $(3.75 \times 13 \mathrm{~mm}$, ASTM Grade $3 \mathrm{CPT}) *$ were divided into two groups of 10 implants each. Ten implants were used as controls and the other 10 implants were used as experimental group with the DLC coating. One implant and one abutment comprised a specimen. For simplicity, the abutment was made from the same materials as the implant (ASTM Grade 3 titanium) and then shaped into cylindrical form by milling procedures (Fig. 1).

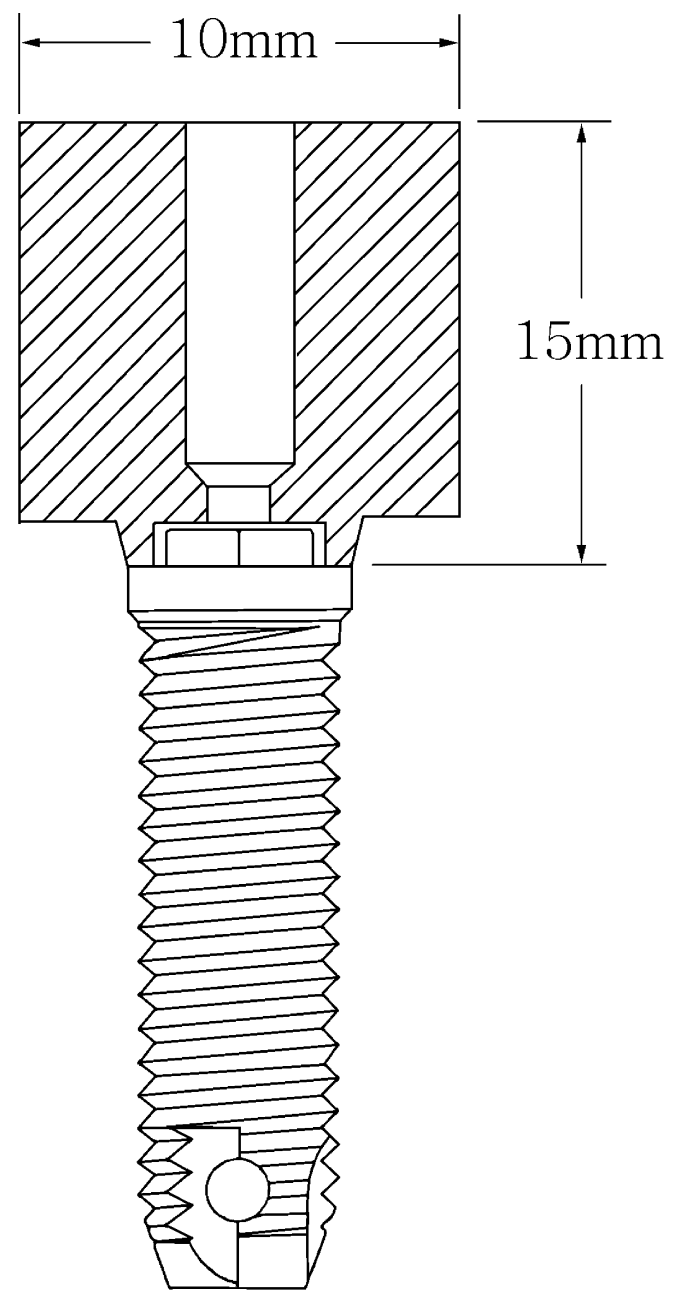

Fig. 1. Diagram of implant-abutment test assembly. A nonsegmental type of abutment was used.

These custom cylindrical, non-segmental (UCLA type) abutments, $15 \mathrm{~mm}$ long with a diameter of $10 \mathrm{~mm}$, replicated the prosthetic element. The abutments had a mean rotational tolerance (misfit) of $3.5^{\circ}$ (Fig. 2). Before tightening the abutment onto the implant with the abutment screw, a DLC coating was deposited onto the experimental group implants.

Silicone-incorporated DLC films $1 \mu \mathrm{m}$ thick were deposited on top of the 10 dental implants (Fig. 3) by 13.56 MHz radio-frequency plasma-assisted chemical vapour deposition (RF PACVD). Briefly, the dental implant underwent the usual wet cleansing procedures prior to coating and was then set on the coating framework that had been designed to expose only the top surface of the implant. The exposed surface was also dry cleansed, using Ar plasma at 3.5 mTorr and a bias 

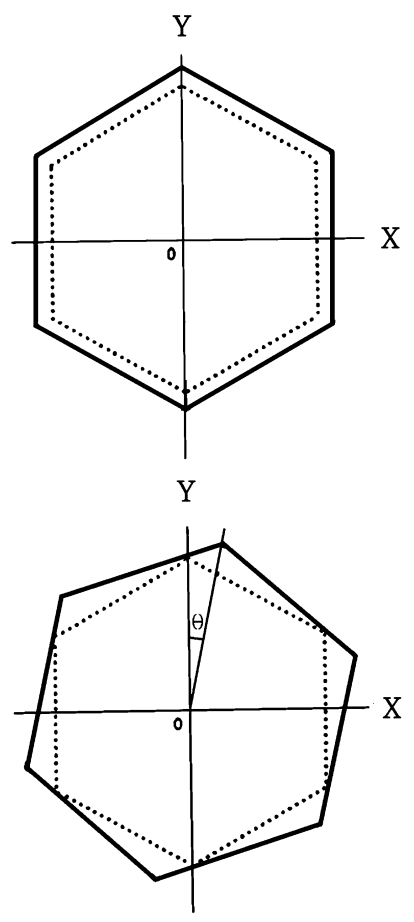

Fig. 2. Rotational misfit of implants. Horizontal cross-sectional view of the hexagon-to-hexagon and abutment-to-implant relationships. The abutment-implant hexagonal relationship was measured as the degree of rotation.

\section{DLC coating surface}

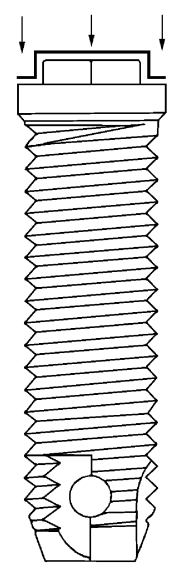

Fig. 3. Diamond Like Carbon (DLC) coating surface. Arrows indicate the surface of the implant receiving the DLC coating.

voltage of $-400 \mathrm{~V}$ for $15 \mathrm{~min}$, prior to coating. After all of these cleansing procedures, a 500-nm thick adhesive silicone layer was applied at a bias voltage of $-200 \mathrm{~V}$ and deposition pressure of 20 mTorr in silane $(10 \%)$ gas diluted with hydrogen gas. The DLC films were coated over this adhesive silicone layer, at 10 mTorr and a bias voltage of $-500 \mathrm{~V}$, to a thickness of $1 \mu \mathrm{m}$ over the top of the dental implants.

\section{Abutment screw loosening test}

The recommended tightening torque, $30 \mathrm{~N}$, was applied to each abutment screw with a torque controller ${ }^{\dagger}$. The abutment screws used in this study were the standard ASTM titanium grade 3 abutment screws ${ }^{\ddagger}$. A custombuilt cyclic loading machine was used, and each sample was subjected to a vertical reciprocal movement on the upper lateral point through a 2-mm diameter stylus. The implants for this study were embedded to the neck position in an acrylic resin (Ortho-Jet Acrylic) ${ }^{\S}$ prior to tightening the abutments.

The cyclic loading machine applied $100 \mathrm{~N}$ of vertical force at a frequency of $20 \mathrm{~Hz}$ to the upper lateral portion of the implant prosthesis until the abutment screws loosened. The cyclic loading machine had a noncontacting displacement sensor (HPT-150) ${ }^{\pi}$ that began each trial with a gap of $0 \cdot 7 \mathrm{~mm}$ between the lateral wall of the implant prosthesis and the tip of the sensor. When the $0 \cdot 7-\mathrm{mm}$ gap was closed, on account of the looseness of the prosthesis because of abutment screw loosening, the cyclic loading procedure automatically stopped and the number of cyclic loadings recorded. After these procedures, the specimens were examined with Scanning Electrone Microscope (S-4700)**.

Statistic analysis for this study consisted of $t$-test and Mann-Whitney test, with $P<0.05$ taken as statistically significant.

\section{Results}

With a force of $100 \mathrm{~N}$ and frequency of $20 \mathrm{~Hz}$, the abutment screw loosening tests were conducted for all DLC-coated and non-coated implants. These data were then compared. One implant in each group suffered a screw fracture before the loosening test could be performed; thus, a total of 18 implants are listed in the results of this study. The data exhibit a significant difference in abutment screw loosening between the coated and non-coated implants. At statistically signi-

\footnotetext{
${ }^{\dagger} 3$, Palm Beach Gardens, FL, USA.

${ }^{\ddagger}$ Soomin Synthesis Dental Materials Co., Seoul, Korea.

${ }^{\S}$ Lang Dental Inc., Wheeting, IL, USA.

${ }^{\mathrm{II}}$ Capacitec. Inc., Ayer, MA, USA.

**Hitachi, Hitachinaka, Japan.
} 
Table 1. Abutment screw loosening for Diamond Like Carbon (DLC)-coated and non-coated groups

\begin{tabular}{lccc}
\hline $\begin{array}{l}\text { DLC coating } \\
\text { implant } \\
\text { number }\end{array}$ & $\begin{array}{l}\text { Loosening } \\
\text { (cycling number) }\end{array}$ & $\begin{array}{l}\text { No coating } \\
\text { Implant } \\
\text { number }\end{array}$ & $\begin{array}{l}\text { Loosening } \\
\text { (cycling number) }\end{array}$ \\
\hline 1 & 46901 & 1 & 14470 \\
2 & 16459 & 2 & 8223 \\
3 & 10847 & 3 & 3846 \\
4 & 37964 & 4 & 2118 \\
5 & 25795 & 5 & 5954 \\
6 & 4000 & 6 & 1376 \\
7 & 16567 & 7 & 4180 \\
8 & 15740 & 8 & 496 \\
9 & 28556 & 9 & 3856 \\
\hline
\end{tabular}

Loosening is reported as the number of loading cycles leading to abutment screw loosening.

Table 2. Statistical analysis of the Diamond Like Carbon (DLC) coating and non-coating groups

\begin{tabular}{lccrlll}
\hline & & & \multicolumn{2}{l}{$P$-values } \\
\cline { 5 - 6 } Group & $n$ & Mean & \multicolumn{1}{c}{ s.d. } & $t$-Test & \multicolumn{2}{l}{ Mann-Whitney } \\
\hline No coating & 9 & 4946.56 & 4271.87 & 0.004 & 0.002 \\
DLC coating & 9 & 22536.56 & 13589.97 & & \\
\hline
\end{tabular}

These data from the screw loosening test indicate that implants with a DLC coating are more resistant to an applied force $(P=0.002)$ than are the non-coated implants.

ficant levels, the implants of the DLC-coating group were more resistant to screw loosening at the force tested here (Tables 1 and 2).

In view of scanning electron microscope of a DLC coated and non coated titanium implant after cyclic loading, the titanium surface is more rough and less resistant than the DLC surface before and after cyclic loading (Fig. 4a,b).

\section{Discussion}

A common complication of single or multiple tooth implant restorations is abutment screw loosening (10). Many attempts have been made to overcome this problem by incorporating anti-rotational and other screw design variations. Lang et al. (11) suggest that the hexagon-to-hexagon orientation, measured as the rotational fit on all abutment systems, was less than the $5^{\circ}$ needed for optimal screw joint stability. In this study, the mean value of the rotational fit was approximately
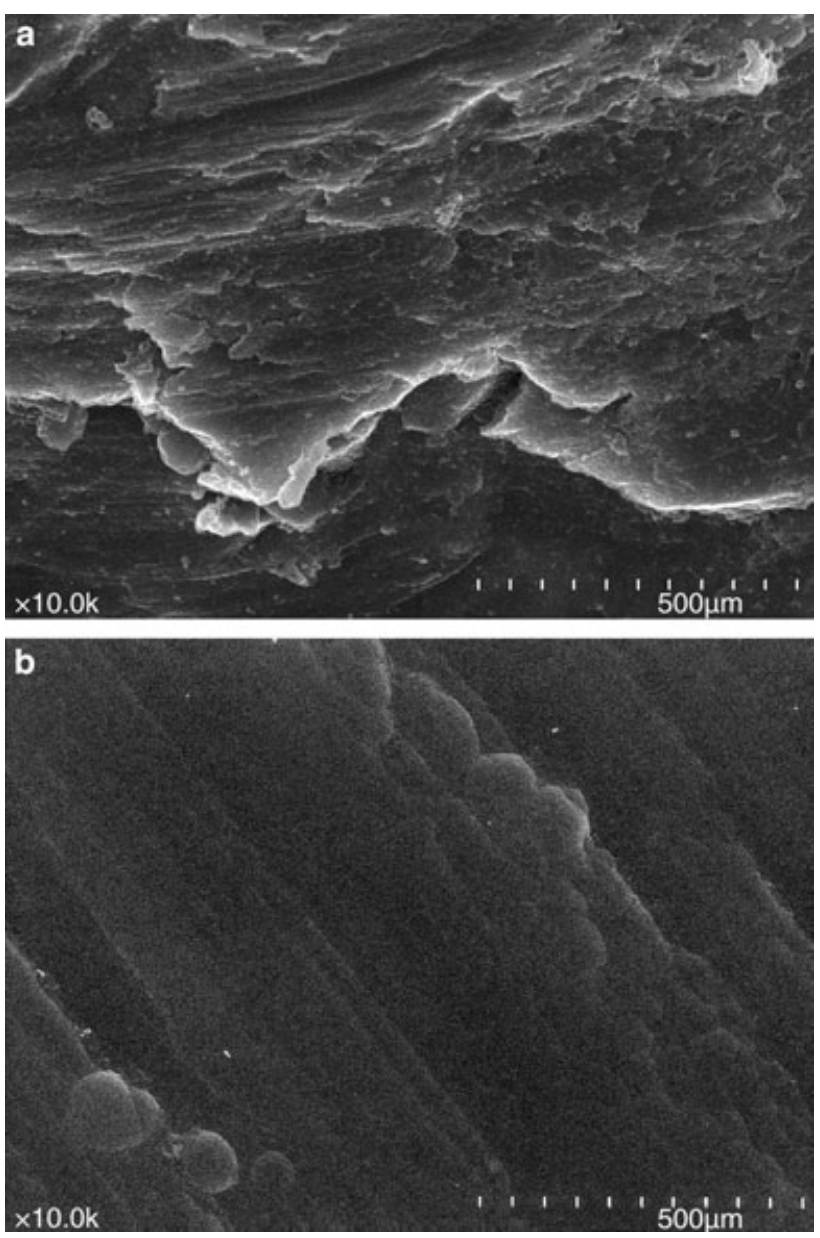

Fig. 4. (a) Scanning electron microscopic view of a non-coated titanium implant after cyclic loading $(\times 10000)$. (b) Scanning electron microscopic view of a Diamond Like Carbon (DLC) coated titanium implant after cyclic loading $(\times 10000)$. The titanium surface is more rough than the DLC surface before and after cyclic loading.

$3.5^{\circ}$ in each group, yet the joint stability of the implants was considered stable.

Screw joint failure occurs in two stages. The first stage comes from an external functional loading that gradually leads to erosion of the pre-load in the screw joint. Any force that causes slippage between the threads releases this stress, and the pre-load becomes evermore diminished (2). It stands to reason that greater frictional forces between the threads require greater external forces for loosening, and this has indeed been exploited as one approach to addressing the problem of screw loosening. Nevertheless, the external load is capable of rapidly eroding whatever preload remains, resulting in micro-movement and subsequent screw loosening. 
The pre-load, precision of fit between the two hexagons, and potential for slippage can also all influence joint stability. In this study, the DLC coating was shown to cause some slippage between the top of the implant and the prosthetic abutment. The DLC layer's lower frictional coefficient than that of pure titanium, and the very hardness of the DLC layer, however, provided some benefit in preventing deformation of the top of the implant. There were observed differences between the two study groups.

Balfour and O'Brien (1) reported that for a lateral force applied to the abutment area of implants including an external hexagon $(0.7 \mathrm{~mm})$, internal octagon, and internal hexagon, the external hexagon implants exhibited the lowest joint failure value. Additionally, the failure mode of the external hexagon implants was exclusive to the physical connection between prosthesis and implants, signifying that the implants could not be restored. The implants' prosthetic connections were also abraded and deformed after the tests of our study. This type of failure is attributable to c.p. titanium implants' soft intrinsic material properties and to their shorter external hexagon prosthetic connections. The DLC film behaved as a protective layer against abrasion or erosion and prevented deformation of the top of the implants as compared with those of the non-coated samples.

The DLC coating on the c.p. titanium surface effectively increases wear resistance. Indeed, the wear resistance of the $1-\mu \mathrm{m}$ DLC layer was much higher in the wear test than was that of the non-coated titanium surface. The superior wear resistance characteristics of the surface were maintained until the DLC layer had worn off. Complementing this wear resistance property, the DLC layer has a peculiarly low friction coefficient, $0 \cdot 2$, that is $50 \%$ lower than the friction coefficient of pure titanium $(0 \cdot 4-0 \cdot 6)(12)$. The high wear resistance and low friction coefficient suggest that a DLC coating can be used in areas of high abrasion. It can, for example, be used in a centrifugal blood pump or ventricular assist device during heart surgery. In these instances, the characteristically low friction coefficient, high wear resistance, and high biocompatibility of DLC are very useful $(7,13)$.

In the present study, the abutment screw loosening between the DLC-coated and non-coated groups showed several differences. Most significantly, the DLC-coated group showed more resistance to screw loosening. The effectiveness of the DLC coating has some relationship to its mechanism of settling on the titanium surface. It may also be due to several other factors, including but not limited to a wedge effect. Further research is most certainly needed so as to broaden understanding of the characteristics of screwloosening on various single tooth implant designs.

\section{Acknowledgments}

This study was supported by a grant from the Korea Health 21 R \& D Project, Ministry of Health \& Welfare, Republic of Korea (01-PJ5-PG1-01CH12-0002).

\section{References}

1. Balfour A, O'Brien GR. Comparative study of antirotational single tooth abutments. Prosthet Dent. 1995;73:36-43.

2. Bickford JH. An introduction to the design and behavior of bolted joints, lst edn. New York: Marcel Dekker Inc; 1995.

3. Sakaguchi RL, Borgersen SE. Nonlinear contact analysis of preload in dental implant screws. Int $\mathrm{J}$ Oral Maxillofac Implants. 1995; 10:295-302.

4. Thull R. Semiconductive properties of passivated titanium and titanium based hard coatings on metals for implants - an experimental approach. Med Prog Technol. 1990;16:225-234.

5. Gil FJ, Solano E, Campos A et al. Improvement of the friction behavior of NiTi orthodontic archwires by nitrogen diffusion. Biomed Mater Eng. 1998;8:335-342.

6. Kim H, Johnson JW. Corrosion of stainless steel, nickeltitanium, coated nickel-titanium, and titanium orthodontic wires. Angle Orthod. 1999;69:39-44.

7. Monties JR, Dion I, Havlik P, Rouais F, Trinkl J, Baquey C. Cora rotary pump for implantable left ventricular assist device: biomaterial aspects. Artif Organs. 1997;21:730-734.

8. Tang L, Tsai C, Gerberich WW, Kruckeberg L, Kania DR. Biocompatibility of chemical-vapour-deposited diamond. Biomaterials. 1995; 16:483-488.

9. Binon PP. Evaluation of the effectiveness of a technique to prevent screw loosening. J Prosthet Dent. 1998;79:430-432.

10. Dixon DL, Breeding LC, Sadler JP, McKay ML. Comparison of screw loosening, rotation, and deflection among three implant designs. J Prosthet Dent. 1995;74:270-278.

11. Lang LA, Wang RF, May KB. The influence of abutment screw tightening on screw joint configuration. J Prosthet Dent. 2002;87:74-79.

12. Kim HW, Koak JY, Lee KR. The abrasion test on DLC coated titanium surface. Korean J Clin Dent. 1999;8:78-81.

13. Yamazaki K, Litwak $\mathrm{P}$, Tagusari $\mathrm{O}$ et al. An implantable centrifugal blood pump with a recirculating purge system (Cool-Seal system). Artif Organs. 1998;22:466-474.

Correspondence: J. Y. Koak, Department of Prosthodontics and Dental Research Institute, College of Dentistry, Seoul National University, Korea.

E-mail: young21c@snu.ac.kr 